\title{
Macrophages steal the show
}

The past decade has seen remarkable advances in tumour immunology due to extensive research on inhibition of programmed cell death protein $1(\mathrm{PD} 1)$ - which is expressed on T cells - and its ligand PDL1, which is expressed on tumour cells. However, little is known about the effect of this pathway in other components of the microenvironment, such as tumour-associated macrophages (TAMs). New findings reveal that macrophages are also important when targeting the PD1PDL1 axis. In one article, Pittet and collaborators show that macrophages can remove anti-PD1 antibodies from $\mathrm{T}$ cells, blunting their response, whereas in another paper, Weissman and colleagues demonstrate that macrophages also express PD1 on their surface, which impairs their phagocytic activity.

To gain insight into the pharmacokinetics of PD1 inhibitors and to investigate potential mechanisms of resistance, Pittet and colleagues followed labelled monoclonal antibodies (mAbs) targeting PD1 ( $a-P D 1)$ in real time and analysed their interaction with different cell types in the tumour

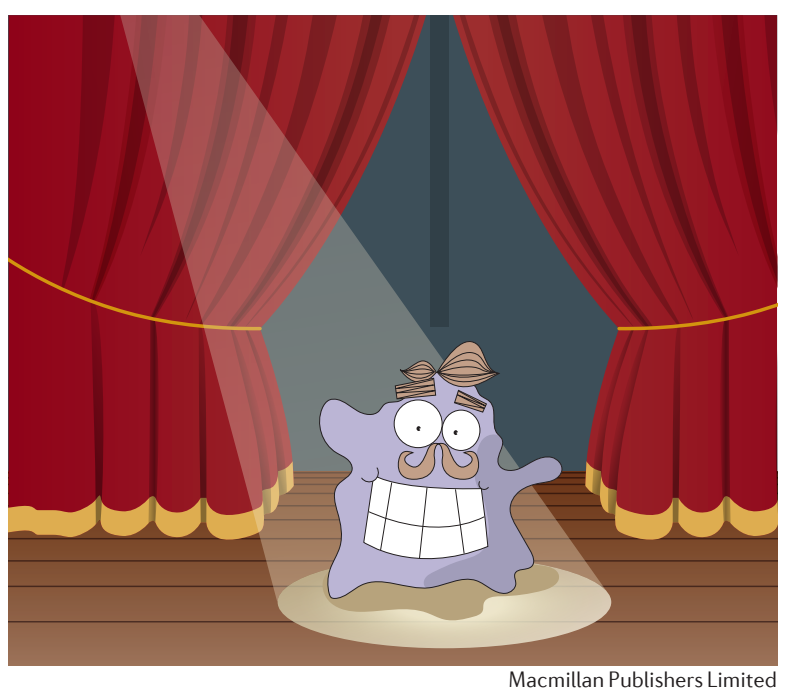

microenvironment in vivo in mice carrying xenografts derived from the MC38 colon cancer cell line. They observed that the drug starts accumulating in tumours within minutes after injection and achieves maximum concentration after $24 \mathrm{~h}$. Reporter mice with labelled $\mathrm{T}$ cells and TAMs showed tagged $\alpha$-PD1 on T cells as early as 5 minutes after injection with a pericellular distribution. However, a-PD1 was then transferred to neighbouring TAMs within minutes, where it was retained. This phenomenon was also observed in mouse models of melanoma and adenocarcinoma.

Further experiments showed that TAMs did not express PD1 on the surface and that the antibody was not sequestered by phagocytosis. Rather, a-PD1 uptake by TAMs depends both on the crystallizable fragment $(\mathrm{Fc})$ domain of the $\mathrm{mAb}$ and on $\mathrm{Fc} \gamma$ receptors (Fc $\gamma \mathrm{Rs})$ expressed by TAMs, and this mechanism was replicated in vitro in human cells treated with the $\mathrm{mAb}$ nivolumab.

Last, the authors assessed the clinical relevance of these findings by inhibiting $\mathrm{Fc} \gamma$ Rs with blocking antibodies. Blockade of Fc $\gamma$ Rs prolonged the presence of $\alpha$-PD1 on T cells and increased the response rate, with complete MC38 tumour rejection achieved in all mice that received the combination treatment.

On the basis of previous results that had reported PD1 expression in macrophages upon infection, Weissman and colleagues wondered whether TAMs might also express PD1. The authors found that $50 \%$ of macrophages isolated from tumours derived from the CT26 colon cancer cell line expressed PD1 on the surface - a number that increased with time after engraftment - whereas no circulating monocytes or splenic macrophages had detectable PD1 expression. Interestingly, the $\mathrm{PD}^{+}$ TAM population began to form around 18-21 days post-engraftment which could explain the differences between the two studies regarding PD1 expression on TAMs. Most of the PD1 ${ }^{+}$TAMs showed a M2-like, pro-tumorigenic profile, whereas the PD1- TAMs had an M1-like, phagocytic phenotype. This pattern was also observed in TAMs from human colorectal tumour samples, with the M2 subset exhibiting higher PD1 expression than the M1 population and being present with increasing frequency as disease progressed. Bone marrow transplantation experiments showed that most PD1+ TAMs originated from circulating leukocytes and not from tumour-resident immune cells.

Next, the authors analysed the effect of PD1 expression on TAM phagocytosis of fluorescent cancer cells in mice lacking T cells. PD1 ${ }^{+}$ TAMs displayed reduced tumour cell phagocytosis compared with $\mathrm{PD}^{-}$ TAMs. Treatment of these mice with mAbs targeting PD1 or PDL1 reduced tumour growth, and these effects were abrogated by TAM depletion, confirming that inhibition of the PD1PDL1 axis in TAMs is responsible for the observed antitumour response. Last, the authors evaluated the effect of inhibiting the anti-phagocytic protein CD47 in combination with a PDL1-targeting $\mathrm{mAb}$, which resulted in greater tumour reduction and increased survival.

These findings add to the growing body of evidence that highlights the importance of macrophages in cancer therapy while offering possibilities to improve the therapeutic success of checkpoint inhibitors.

M. Teresa Villanueva

This article originally appeared in Nat. Rev. Cancer (http://dx.doi.org/10.1038/nrc.2017.47)

ORIGINAL ARTICLES Arlauckas, S. P. et al. In vivo imaging reveals a tumor-associated macrophagemediated resistance pathway in anti-PD-1 therapy. Sci. TranslMed. 9, eaal3604 (2017)| Gordon, S. R. et al. PD-1 expression by tumourassociated macrophages inhibits phagocytosis and tumour immunity. Nature, 545, 495-499 (2017) 